\title{
Block transitive Steiner systems with more than one point orbit
}

\author{
David M. Evans
}

\begin{abstract}
For all 'reasonable' finite $t, k$ and $s$ we construct a $t$ - $\left(\aleph_{0}, k, 1\right)$ design and a group of automorphisms which is transitive on blocks and has $s$ orbits on points. In particular, there is a $2-\left(\aleph_{0}, 4,1\right)$ design with a block-transitive group of automorphisms having two point orbits. This answers a question of P. J. Cameron and C. E. Praeger. The construction is presented in a purely combinatorial way, but is a byproduct of a new way of looking at a model-theoretic construction of E. Hrushovski.

2000 Mathematics Subject Classification: 05B05 (Primary); $20 \mathrm{~B} 27$ (Secondary)
\end{abstract}

By a $t$ - $(v, k, \lambda)$ design we mean a set $P$ of points, of cardinality $v$, together with a set $\mathcal{B}$ of blocks each of which is a $k$-subset of $P$, and which has the property that any set of $t$ points is a subset of exactly $\lambda$ blocks. An automorphism of the design $(P, \mathcal{B})$ is simply a permutation of $P$ which preserves $\mathcal{B}$. It is well known that if $G$ is a group of automorphisms of the design $(P, \mathcal{B})$ and $t \geq 2$ and $v, k, \lambda$ are finite, then the number of $G$-orbits on $P$ is no greater than the number of $G$-orbits on $\mathcal{B}$ : this is commonly referred to as Block's Lemma ([1]).

For the rest of the paper we suppose $t \geq 2, v$ is infinite and $t, k, \lambda$ are finite. A standard model-theoretic trick (cf. [2], Section 2.2) shows that the actual infinite cardinality $v$ is irrelevant to our concerns and we will emphasise this by referring to ' $t-(\infty, k, \lambda)$ designs.' However, in the designs we construct $P$ will be countably infinite, so $v$ is actually $\aleph_{0}$.

It is known that Block's Lemma need not hold for $t-(\infty, k, \lambda)$ designs. In unpublished work from 1995, the Author used ideas from [7] to construct a $2-\left(\aleph_{0}, 4,14\right)$ design admitting a group of automorphisms with one block 
orbit and two point orbits. Based on a private communication, his method was subsequently extended by Camina and Webb ([4], [8]) to produce other examples of the failure of Block's Lemma. However, what is possibly the most interesting case, that of Steiner systems, where $\lambda=1$, proved elusive. It is this which we address in this paper.

Suppose $(P, \mathcal{B})$ is a $t$ - $(\infty, k, 1)$ design and $G$ is a group of automorphisms which is transitive on $\mathcal{B}$. Let $C_{1}, \ldots, C_{s}$ denote the $G$-orbits on points. Then any block contains the same number $n_{i}$ of points of $C_{i}$. So of course, as any $t$-set is in a unique block, if we disregard the case where some $C_{i}$ has fewer than $t$ points, we must have that $n_{i} \geq t$ for all $i$. As $n_{1}+\cdots+n_{s}=k$, this means that $s \leq k / t$. Thus, the smallest case where we might have $s>1$ is a $2-(\infty, 4,1)$ design and $G$ block transitive with 2 point orbits. In this case, each block contains exactly 2 points from each orbit. The existence of such a design was a question posed by Peter Cameron and Cheryl Praeger at the British Combinatorial Conference in 1993 [3]. The following, which answers this question affirmatively, is the main result of this paper.

Theorem 1 Suppose $t \geq 2$, and $k, s$ are finite with $s \leq k / t$ and $k>t$. Then there exists a $t-(\infty, k, 1)$ design $(P, \mathcal{B})$ and a group $G$ of automorphisms such that $G$ is transitive on $\mathcal{B}$ and has s orbits on $P$. Moreover, if natural numbers $n_{1}, \ldots, n_{s}$ satisfy $n_{i} \geq t$ and $n_{1}+\cdots+n_{s}=k$, then we can ensure that each block contains $n_{i}$ points of the $i$-th point orbit.

The remainder of the paper is a sequence of lemmas culminating in a proof of this result. Henceforth, the parameters $t, k, s, n_{1}, \ldots, n_{s}$ are fixed and satisfy the hypotheses of Theorem 1 . For the construction of the design $(P, \mathcal{B})$ in the theorem, we work with finite structures consisting of a set $A$ and a set $E_{A}$ of $k$-subsets of $A$. We refer to the elements of $A$ as the points of the structure and the elements of $E_{A}$ as the edges of the structure: these structures are not designs, so we prefer to use the terminology of $k$ uniform hypergraphs, but say 'edge' rather than 'hyperedge.' Each point of the structure will have exactly one of $s$ colours $c_{1}, \ldots, c_{s}$ (- corresponding to the orbits in the eventual infinite structure) and each edge contains $n_{i}$ points of colour $c_{i}$. We refer to these types of structures as unoriented coloured hypergraphs. So formally an unoriented coloured hypergraph consists of a triple $\left(A, E_{A}, c\right)$ where $c: A \rightarrow\left\{c_{1}, \ldots, c_{s}\right\}$ is the function describing the colouring. However, we shall usually just denote this by $A$, rather than the triple. 
In an orientation of a coloured hypergraph $\left(A, E_{A}, c\right)$ we distinguish $k-t$ points of every edge $e$, and call each of them an apex of $e$. So formally we can think of an orientation as being a function $w: E_{A} \times A \rightarrow\{0,1\}$ where, for each $e \in E_{A}$ we have $w(e, a)=1$ for precisely $k-t$ points $a$, all of which lie in $e$ (the apices of $e$ ). An oriented coloured hypergraph is then a quadruple $\left(A, E_{A}, c, w\right)$, where $w$ is an orientation of the coloured hypergraph $\left(A, E_{A}, c\right)$. To explain the terminology it might help to consider what for us is a degenerate case. Suppose $k=2$, so we are dealing with ordinary graphs, and $t=1$. Thus for an orientation we are required to select one point from every edge. This is exactly what we do in a directed graph: regard the direction on the edge as being away from the selected point.

In both the oriented and unoriented cases, if we have such a structure $B$ and $A \subseteq B$ then we regard $A$ as a structure by giving its points the colours inherited from $B$, and taking the edges of $B$ which are contained in $A$ (with the same apices, in the oriented case).

Definition 1 Let $\mathcal{G}$ denote the class of finite oriented coloured hypergraphs as above which satisfy:

(i) each point is an apex of at most one edge;

(ii) two edges have at most $t-1$ points in common.

We let $\mathcal{G}_{0}$ denote the larger class just satisfying (i).

If $A \subseteq B \in \mathcal{G}$, then we write $A \sqsubseteq B$ and say that $A$ is closed in $B$ to mean that if $e$ is an edge of $B$ and some apex of $e$ is in $A$, then $e \subseteq A$.

Definition 2 Let $\mathcal{C}$ be the class of all finite coloured hypergraphs which have a $\mathcal{G}$-orientation i.e. an orientation in $\mathcal{G}$. (In other words, $\mathcal{C}$ consists of the structures in $\mathcal{G}$ where we forget which vertices are apices.) Similarly $\mathcal{C}_{0}$ consists of finite coloured hypergraphs having an orientation in $\mathcal{G}_{0}$.

If $A \subseteq B \in \mathcal{C}$, then we write $A \leq B$ to mean that there is a $\mathcal{G}$-orientation of $B$ in which $A$ is closed.

We outline the connection with Hrushovski's construction from [7]. If $B$ is a finite (coloured) hypergraph then we let $\delta(B)=|B|-(k-t)\left|E_{B}\right|$. Write $A \leq{ }^{*} B$ iff for all $A \subseteq B_{1} \subseteq B$ we have $\delta(A) \leq \delta\left(B_{1}\right)$. The folowing lemma is from [6], but for the convenience of the reader we sketch a slightly different proof here.

Lemma 1 If $B$ is a finite (coloured) hypergraph and $A \subseteq B$, then there is a $\mathcal{G}_{0}$-orientation of $B$ in which $A$ is closed if and only if $\emptyset \leq^{*} A \leq^{*} B$. 
Proof. Suppose there is a $\mathcal{G}_{0}$-orientation of $B$ in which $A$ is closed. Then a simple counting argument shows that $\delta(A) \leq \delta(B)$. As the same is true for any substructure of $B$ which contains $A$, we therefore obtain $A \leq{ }^{*} B$. The $\mathcal{G}_{0}$-orientation on $B$ induces a $\mathcal{G}_{0}$-orientation of $A$ in which $\emptyset$ is obviously closed, so from this we also obtain $\emptyset \leq^{*} A$.

Suppose conversely that $\emptyset \leq^{*} A \leq{ }^{*} B$. Arguing inductively, we may assume that we already have a $\mathcal{G}_{0}$-orientation of $A$. We want to extend this to a $\mathcal{G}_{0}$-orientation of $B$ in which $A$ is closed. Thus, for each edge in $E_{B} \backslash E_{A}$ we wish to select $k-t$ of its points to be its apices. Note that as $A$ is to be closed in $B$, these points should be in $B \backslash A$. Moreover, each point of $B \backslash A$ should be an apex of at most one edge.

Consider the following bipartite graph $\Gamma$. As vertices we have $\Gamma_{1}=\left(E_{B} \backslash E_{A}\right) \times\{1, \ldots, k-t\}$ and $\Gamma_{2}=B \backslash A$. For the edges, we say that a pair $(e, i) \in \Gamma_{1}$ is adjacent to $b \in \Gamma_{2}$ iff $b \in e$. We show that this has a complete matching from $\Gamma_{1}$ to $\Gamma_{2}$. Indeed, let $X \subseteq \Gamma_{1}$ and let $\Gamma(X)$ denote the set of vertices in $\Gamma_{2}$ adjacent to some vertex in $X$. Let $Y=$ $\bigcup\{e: \exists i(e, i) \in X\}$. So $Y$ consists of points of $B$ contained in edges of $B$ represented in $X$. So $\Gamma(X)=Y \backslash Y \cap A$. Moreover $|X| \leq(k-t)\left|E_{Y} \backslash E_{Y \cap A}\right|$, just by definition of $\Gamma$.

Now, as $A \leq^{*} B$ we have $A \cap Y \leq^{*} Y$ (this is a standard property of such notions and we do not reproduce the proof here). So $\delta(A \cap Y) \leq$ $\delta(Y)$. Together with the previous paragraph, this gives $|X| \leq \Gamma(X)$, which is exactly the condition we need to apply Philip Hall's Marriage Theorem to $\Gamma$. Hence, there is a complete matching of $\Gamma$ from $\Gamma_{1}$ to $\Gamma_{2}$, as required.

To obtain an orientation of $B$, we simply say that if $e \in E_{B} \backslash E_{A}$ and $b \in e$, we make $b$ an apex of $e$ whenever there is $i \leq k-t$ such that $\{(e, i), b\}$ is in the matching.

Thus, the notions $\leq^{*}$ and $\leq$ coincide. Our approach here is really the same as that using Hrushovski's method, but the presentation seems to us to be considerably simpler and allows us to isolate the parts of the construction we really need.

In what follows, we generally suppress the adjective 'coloured.'

Lemma 2 (1) If $B \in \mathcal{G}$ and $A \sqsubseteq B$ and $C \subseteq B$, then $A \cap C \sqsubseteq C$.

(2) If $A \sqsubseteq B \sqsubseteq C \in \mathcal{G}$ then $A \sqsubseteq C$.

Proof. (1) Suppose an edge $e \subseteq C$ has an apex $a \in A$. As $A \sqsubseteq B$ we have $e \subseteq A$, so $e \subseteq A \cap C$, as required. 
(2) Take an edge $e$ of $C$ which has an apex $a$ in $A$. Then $a \in B$ and as $B \sqsubseteq C$ this implies $e \subseteq B$. As $A \sqsubseteq B$ we then get $e \subseteq A$, as required.

For the following, recall that the class $\mathcal{C}$ of unoriented coloured hypergraphs is defined in Definition 2.

Lemma 3 (1) If $A \leq B \in \mathcal{C}$ then any $\mathcal{G}$-orientation of $A$ extends to a $\mathcal{G}$-orientation of $B$.

(2) If $B \in \mathcal{C}$ and $A \leq B$ and $C \subseteq B$, then $A \cap C \leq C$.

(3) If $A \leq B \leq C \in \mathcal{C}$, then $A \leq C$.

Proof. (1) As $A \leq B \in \mathcal{C}$ there is some $\mathcal{G}$-orientation $B^{\prime}$ of $B$ in which $A$ is closed. Suppose we are given a $\mathcal{G}$-orientation $A^{\prime}$ of $A$. In $B^{\prime}$ change the orientation on edges in $A$ to the orientation given by $A^{\prime}$. Call the result $B^{\prime \prime}$. Note that $A \sqsubseteq B^{\prime \prime}$. We claim that $B^{\prime \prime} \in \mathcal{G}$. It is clear that $B^{\prime \prime}$ is an oriented hypergraph and so we need to check (i), (ii) in Definition 1. Now, (ii) is clear as this is just a property of the (unoriented) hypergraph structure on $B$, and we did not change this. For (i), suppose $x$ is an apex of edges $e_{1}, e_{2}$ of $B^{\prime \prime}$. If $x \in A$ then $e_{1}, e_{2} \subseteq A$ as $A \sqsubseteq B^{\prime \prime}$, so as $A^{\prime} \in \mathcal{G}$ we get $e_{1}=e_{2}$. But if $x \notin A$, then $e_{i} \nsubseteq A$ so the apices of $e_{1}, e_{2}$ are the same in $B^{\prime}, B^{\prime \prime}$. As $B^{\prime} \in \mathcal{G}$ it follows that $e_{1}=e_{2}$.

(2) Take a $\mathcal{G}$-orientation of $B$ in which $A$ is closed and apply Lemma 2(1).

(3) Take a $\mathcal{G}$-orientation of $C$ in which $B$ is closed. In this, exchange the orientation on $B$ for a $\mathcal{G}$-orientation of $B$ in which $A$ is closed. By (1) (or rather, its proof), the result is still a $\mathcal{G}$-orientation of $C$, and in it, $A$ is closed.

Definition 3 Let $\mathcal{C}_{1}$ consist of all $B \in \mathcal{C}$ such that for every $X \subseteq B$ with $|X| \leq t$ we have $X \leq B$. Let $\mathcal{G}_{1}$ be the class of all $\mathcal{G}$-orientations of these.

Remarks 1 By definition, both of these classes are closed under substructures and Lemmas 2 and 3 also hold for these classes. It is useful to see this extra condition on $\mathcal{C}_{1}$ in terms of the function $\delta$. If $X$ has size at most $t$ then $\delta(X)=|X|$. So by Lemma 1 , the condition that $X \leq B$ is equivalent to saying that $\delta(Y) \geq|X|$ for all $Y \subseteq B$ which contain $X$. In other words, if $B \in \mathcal{C}$, then $B \in \mathcal{C}_{1}$ iff $\delta(Y) \geq \min (|Y|, t)$ for all $Y \subseteq B$.

Definition 4 Suppose $B_{1}, B_{2} \in \mathcal{G}$ have a common substructure $A$ and $A \sqsubseteq$ $B_{1}, B_{2}$. The free amalgam $F$ of $B_{1}$ and $B_{2}$ over $A$ has point set equal to the 
disjoint union of $B_{1}$ and $B_{2}$ over $A$ and the points of $F$ have the same colours as in $B_{1}, B_{2}$. The edges of $F$ are the edges of $B_{1}$ and those of $B_{2}$, together with the same apices as in these structures.

In the above defintion, there is no ambiguity about colours of points and apices of edges contained in $A$ as $A$ is assumed a common substructure of $B_{1}$ and $B_{2}$. It is easy to see that $F$ is an oriented coloured hypergraph and $B_{i} \sqsubseteq F$. Moreover every point is an apex of at most one edge of $F$. Indeed, if the point is in $B_{i} \backslash A$, then any edge of which it is an apex is contained in $B_{i}$ as $B_{i} \sqsubseteq F$; if it is in $A$ then every edge of which it is an apex is contained in $A$. Thus (in the notation of Definition 1 ), $F \in \mathcal{G}_{0}$.

Lemma 4 (Б-amalgamation lemma) Suppose $B_{1}, B_{2} \in \mathcal{G}_{1}$ have a common substructure $A$ and $A \sqsubseteq B_{1}, B_{2}$. Then there exists $D \in \mathcal{G}_{1}$ and embeddings $f_{i}: B_{i} \rightarrow D$ such that $f_{i}\left(B_{i}\right) \sqsubseteq D$ and $f_{1}\left|A=f_{2}\right| A$.

By 'embedding' we mean that $f_{i}$ is an isomorphism between $B_{i}$ and the oriented coloured hypergraph structure on $f_{i}\left(B_{i}\right)$ induced from $D$.

Proof. This is by induction on $\left|B_{1} \backslash A\right|+\left|B_{2} \backslash A\right|$.

Let $F$ be the free amalgam of $B_{1}$ and $B_{2}$ over $A$. So by the above, we know that $F \in \mathcal{G}_{0}$. We now show that if $X \subseteq F$ has at most $t$ points, then $X \leq F$. Note first that as $t<k$, we have $\delta(X)=|X| \leq t$. We shall now use the observations in Remarks 1. Let $X \subseteq Y \subseteq F$ and $Y_{i}=Y \cap B_{i}$ (for $i=1,2)$ and $Y_{0}=Y \cap A$. Then $Y$ is the free amalgam of $Y_{1}$ and $Y_{2}$ over $Y_{0}$ so $\delta(Y)=\delta\left(Y_{1}\right)+\delta\left(Y_{2}\right)-\delta\left(Y_{0}\right)$. If $\left|Y_{i}\right| \leq t$ (for $\left.i=1,2\right)$, then $\delta\left(Y_{i}\right)=\left|Y_{i}\right|$ and $\delta\left(Y_{0}\right)=\left|Y_{0}\right|$. Thus if $\left|Y_{1}\right|,\left|Y_{2}\right| \leq t$, then $\delta(Y)=|Y| \geq|X|=\delta(X)$. So suppose $\left|Y_{2}\right| \geq t$. Then $\delta\left(Y_{2}\right) \geq t$ (as $B_{2} \in \mathcal{G}_{1}$ ), and as $\delta\left(Y_{1}\right) \geq \delta\left(Y_{0}\right)$ (because $Y_{0} \leq Y_{1}$ ) we get $\delta(Y) \geq t$, so $\delta(Y) \geq \delta(X)$, as required.

If $F \in \mathcal{G}_{1}$ then we can take $D=F$. If not, then by the above and defintion of $\mathcal{G}_{1}$ there exist edges $e_{1}, e_{2}$ of $F$ which have at least $t$ points in common. These cannot both be contained in $B_{2}$, so without loss $e_{1} \cap B_{1} \backslash A$ is non-empty. This means that all apices of $e_{1}$ are in $B_{1} \backslash A$ (as $A \sqsubseteq F$ ) and also that $e_{1} \subseteq B_{1}$ (as $\left.B_{1} \sqsubseteq F\right)$. Thus $e_{2} \nsubseteq B_{1}$, so a similar argument shows that all apices of $e_{2}$ must be in $B_{2} \backslash A$ and $e_{2} \subseteq B_{2}$. As any edge has $t$ non-apices, it follows that $e_{1} \cap e_{2}=e_{i} \cap A$ and that the apices of $e_{i}$ are precisely those points of $e_{i}$ outside $A$.

Let $A_{i}^{\prime}=A \cup e_{i} \subseteq B_{i}$. Now, note that if $x \in A_{i}^{\prime}$ then any edge of $B_{i}$ of which $x$ is an apex must be contained in $A_{i}^{\prime}$ : if $x \notin A$ the edge must be $e_{i}$; if 
$x \in A$ this is automatic as $A \sqsubseteq B_{i}$. Thus $A_{i}^{\prime} \sqsubseteq B_{i}$. Moreover, there are no edges of $B_{i}$ contained in $A_{i}^{\prime}$ apart from $e_{i}$ and the edges of $A$ : consider what an apex of such an edge could be. It follows that there is an isomorphism between $A_{1}^{\prime}$ and $A_{2}^{\prime}$ (as substructures of $B_{1}, B_{2}$ ) which is the identity on $A$. Thus we can regard $A_{1}^{\prime}$ as a common substructure of both $B_{1}$ and $B_{2}$, closed in each, and apply the inductive hypothesis to obtain $D$.

Corollary 1 ( $\leq$-amalgamation lemma) Suppose $B_{1}, B_{2} \in \mathcal{C}_{1}$ have a common substructure $A$ and $A \leq B_{1}, B_{2}$. Then there exists $D \in \mathcal{C}_{1}$ and embeddings $f_{i}: B_{i} \rightarrow D$ such that $f_{i}\left(B_{i}\right) \leq D$ and $f_{1}\left|A=f_{2}\right| A$.

Proof. Take a $\mathcal{G}$-orientation $B_{1}^{\prime}$ of $B_{1}$ in which $A$ is closed. So by definition, $B_{1}^{\prime} \in \mathcal{G}_{1}$. By Lemma $3(1)$, the induced orientation on $A$ extends to a $\mathcal{G}$-orientation $B_{2}^{\prime}$ of $B_{2}$ in which $A$ is closed. We can use Lemma 4 to find $D^{\prime} \in \mathcal{G}_{1}$ into which $B_{1}^{\prime}$ and $B_{2}^{\prime}$ embed over $A$ as closed substructures. Forgetting the orientation gives us $D \in \mathcal{C}_{1}$, as required.

Now that we have Lemma 3 and Corollary 1, there is a standard construction, sometimes known as the Fraïssé limit, which allows us to build a countably infinite structure with a rich automorphism group from the finite structures in $\mathcal{C}_{1}$. In the proof of Corollary 2 below, we shall give a sketch of the construction, applied to our particular case. A more detailed exposition of the construction can be found, for example, in ([5], Section 2.1.3). Before doing this, we want to extend the class $\mathcal{C}_{1}$ and the relation $\leq$ to include infinite structures. We consider an infinite structure $M$ all of whose finite substructures are in $\mathcal{C}_{1}$. If $A$ is a finite substructure of $M$ then we write $A \leq M$ to mean that $A \leq B$ for all finite $B \subseteq M$ with $A \subseteq M$.

Corollary 2 There exists a countably infinite unoriented coloured hypergraph $M$ with the properties that:

(1) $M$ is the union of a chain $M_{1} \leq M_{2} \leq M_{3} \leq \cdots$ of finite substructures $M_{i}$, each in $\mathcal{C}_{1}$, and with $M_{i} \leq M_{i+1}$ for all $i \in \mathbb{N}$ (where $\mathbb{N}$ denotes the set of natural numbers);

(2) if $A \leq M$ is finite and $A \leq B \in \mathcal{C}_{1}$, then there exists an embedding $f: B \rightarrow M$ with $f(B) \leq M$ and $f(a)=a$ for all $a \in A$.

Moreover, if $X_{1}, X_{2} \leq M$ are finite and $g: X_{1} \rightarrow X_{2}$ is an isomorphism between the induced substructures, then these exists an automorphism of $M$ which extends $g$. 
Proof. Note that by transitivity of $\leq$ (Lemma 3) it is enough to find a chain $M_{1} \leq M_{2} \leq M_{3} \leq \ldots$ of finite structures in $\mathcal{C}_{1}$ such that for all $i$ and $B \in \mathcal{C}_{1}$, if $A \leq M_{i}$ and $A \leq B$ then there is $j \geq i$ and an embedding $f: B \rightarrow M_{j}$ with $f(B) \leq M_{j}$ which is the identity on $A$ (for then we take $M$ to be the union of the $M_{i}$ ). We can do this inductively as follows.

Take $M_{1}$ to be anything in $\mathcal{C}_{1}$. Suppose we have constructed $M_{1} \leq \cdots \leq$ $M_{i}$. We will construct $M_{i+1}$ so that if $A \leq M_{i}$ and $A \leq B \in \mathcal{C}_{1}$ and $|B| \leq i$, then there is an embedding $f: B \rightarrow M_{i+1}$ which is the identity on $A$ and has $f(B) \leq M_{i+1}$. Note that, for fixed $i$, there are essentially only finitely many possibilities for the pairs $A \leq B$ here: $\left(A_{1}, B_{1}\right), \ldots,\left(A_{r}, B_{r}\right)$, say. Using Corollary 1 , we can amalgamate $M_{i}$ and $B_{1}$ over the common $\leq-$ substructure $A_{1}$, to obtain $M_{i}^{1} \in \mathcal{C}_{1}$ with $M_{i}, B_{1} \leq M_{i}^{1}$ (strictly speaking $B_{1}$ is only isomorphic to a $\leq$-substructure of $M_{i}^{1}$, but it is harmless to identify this substructure with $B_{1}$ ). By Lemma $3, A_{2} \leq M_{i}^{1}$, so we can repeat the argument to obtain $M_{i}^{2} \in \mathcal{C}_{1}$ with $M_{i}^{1}, B_{2} \leq M_{i}^{2}$. We continue in this way for each of the pairs, and take $M_{i+1}=M_{i}^{r}$.

The 'moreover' part is a standard back-and-forth argument, and again we only sketch the details. The key point is that given an isomorphism $h$ between finite $\leq$-substructures $X, Y$ of $M$, and given $a \in M$, we can find finite $X^{\prime}, Y^{\prime} \leq M$ with $X^{\prime}$ containing $X, a$ and $Y^{\prime}$ containing $Y$, and an isomorphism $h^{\prime}: X^{\prime} \rightarrow Y^{\prime}$ extending $h$. To do this, note that condition (1) guarantees that we can find a finite $X^{\prime} \leq M$ which contains $X$ and $a$. Using $h^{-1}$, there is an isomorphic copy $Y^{\prime} \in \mathcal{C}_{1}$ of $X^{\prime}$ which contains $Y$. Using (2), we may regard $Y^{\prime}$ as a $\leq$-substructure of $M$.

We can now finally return to designs and give the proof of the main result.

Proof of Theorem 1. Let $M$ be as in Corollary 2 and $G$ the automorphism group of $M$. So $G$ consists of the permutations of the set $M$ which preserve the set of edges and the colours of points. Take the points of $M$ to be the points, and the edges of $M$ to be the blocks of our design. By Definition 2, if $X \subseteq M$ has size $t$, then there is at most one block containing $X$. On the other hand $X \leq M$ (by definition of $\mathcal{C}_{1}$ ) and $X \leq B$, where $B \in \mathcal{C}_{1}$ is a $k$-element structure in which all points lie in a single edge and there are $n_{i}$ points of $B$ of colour $c_{i}$. So by (2) in Corollary 2 there is an embedding of $B$ into $M$ which is the identity on $X$ and whose image is $\leq M$. In other words, there is a block in $M$ which contains $X$ and this block is $\leq M$. Thus any $t$ points of $M$ are contained in a unique block, so we have a $t-\left(\aleph_{0}, k, 1\right)$ design.

By the previous paragraph, any block is $\leq M$. So by the 'moreover' part 
of Corollary 2, $G$ is transitive on the set of blocks, and on the set of points of any particular colour. Thus the $G$-orbits on points are described by the colours, as required.

\section{References}

[1] R. E. Block, 'On the orbits of collineation groups', Mathematische Zeitschrift 96 (1967), 33-49.

[2] Peter J. Cameron, Oligomorphic Permutation Groups, London Math. Soc. Lecture Notes Series 152, Cambridge University Press, Cambridge, 1990.

[3] Peter J. Cameron and Cheryl E. Praeger, Proposed problem at the 14th British Combinatorial Conference. Problem 227 of 'Research Problems', Discrete Mathematics 138 (1995), 405-411.

[4] Alan R. Camina, 'Block-transitive, point-intransitive block designs', in Combinatorial Designs and their Applications Research Notes in Mathematics Series, 403 (ed. F. C. Holroyd, K. A. S. Quinn, C. Rowley, B. S. Webb), pp. 71-82, Chapman and Hall,1999.

[5] David M. Evans, 'Examples of $\aleph_{0}$-categorical structures', in Automorphisms of First-Order Structures (edited by Richard Kaye and Dugald Macpherson), pp 33-72, Oxford Science Publications, Oxford, 1994.

[6] David M. Evans, 'Trivial stable structures with non-trivial reducts', Preprint, Norwich, 2003.

[7] Ehud Hrushovski, 'A new strongly minimal set', Annals of Pure and Applied Logic 62 (1993), 147-166.

[8] Bridget S. Webb, 'Designs with more point-orbits than block-orbits', Ars Combinatoria 53 (1999), 291-308.

Author's address:

School of Mathematics, UEA, Norwich NR4 7TJ, England

e-mail: d.evans@uea.ac.uk

3 February 2004. 